\title{
Tecnologías de control de floraciones de cianobacterias y algas nocivas en cuerpos de agua, con énfasis en el uso de irradiación por ultrasonido
}

\author{
Technologies for the control of harmful cyanobacteria \\ and algal blooms in waterbodies, with emphasis in the use \\ of ultrasound irradiation
}

\author{
Míguez, Diana (1) \\ (1) Programa Aguas, Proyectos Ambientales, Gerencia I+D+i, Laboratorio Tecnológico del Uruguay, LATU. \\ Contacto:dmiguez@latu.org.uy \\ RECIBIDO: 21/11/2016 - APROBADO: 21/12/2016
}

\begin{abstract}
Resumen
Esta revisión sintetiza las ventajas y desventajas de los métodos de control de floraciones de cianobacterias y algas nocivas y/o de sus toxinas en cursos de agua o en agua potable. En los últimos años, las floraciones, o sea, el crecimiento desmedido de cianobacterias, han aumentado en extensión y frecuencia en el mundo y los riesgos ambientales y sanitarios por la potencial producción de toxinas. Además, la concomitante disminución de los niveles de oxígeno disuelto en las aguas afecta los ecosistemas, implicando a veces la mortandad de peces. Los métodos utilizados para el control y la remediación de las floraciones incluyen: los químicos, para destruir las células, o disminuir los niveles de fósforo y otros nutrientes en agua y en sedimentos; los biológicos, basados en la relación predador-presa, y los físicos, que atacan la estructura celular, su flotabilidad o viabilidad. Entre estos últimos se incluye la tecnología de ultrasonido, que también puede involucrar la generación de radicales libres. Este último método es promisorio, pero requiere continuar investigando para evaluar su eficiencia e inocuidad. También se hace referencia a los proyectos emprendidos en el marco del Programa Aguas LATU/Latitud, utilizando irradiación por ultrasonido en un tajamar y un estanque eutrofizados.

Palabras clave: Floraciones de algas y cianobacterias nocivas, ultrasonido, eutrofización, restauración de lagos, ecotoxicidad.
\end{abstract}

\begin{abstract}
This review summarizes the advantages and disadvantages of methods of control of harmful algal and cyanobacterial blooms and/or their toxins in watercourses or in drinking water. In recent years, the blooming phenomena, or the excessive growth of cyanobacteria, have increased in extent and frequency around the world, involving increasingly higher environmental and health risks due to the potential production of toxins. In addition, the concomitant decrease in the levels of dissolved oxygen in waters cause effects on the ecosystems that could even lead to fish mortality events. The methods used to control and remediate the blooms range from chemicals to destroy the cells or they focus on reducing the concentration of phosphorus and other nutrients in the water and sediments, to biological based on predator-prey relationships, and to physical methods, aimed to attack the cell structure, affect its buoyancy or their viability, including among them the ultrasound technology, of promising applicability, but requiring further research to evaluate its efficiency and safety. A reference is made to research projects undertaken in the framework of the LATU/Latitud Water Program, by applying ultrasound irradiation in an eutrophic excavated lagoon and in a pond. Keywords: Harmful algal and cyanobacterial blooms, ultrasound, eutrophication, lake restoration, ecotoxicity.
\end{abstract}




\title{
Tabla de contenidos
}

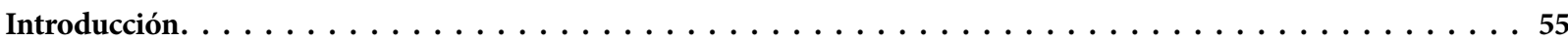

Prevalencia mundial de las floraciones de cianobacterias nocivas y de los brotes de toxicidad . . . . . . . . . . . . . . . . . . . . . . . . . . Antecedentes de eutrofización y floraciones de algas y cianobacterias nocivas en Uruguay. . . . . . . . . . . . . . . . 56

\begin{abstract}
Métodos 57

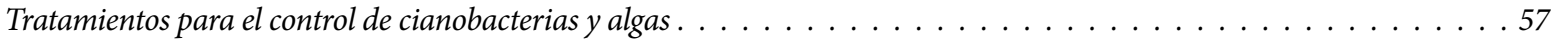

Tratamientos de remoción de las cianotoxinas . . . . . . . . . . . . . . . . . . . . . . . . . . . . . . 57

Características de la tecnología de ultrasonido y sus mecanismos de acción . . . . . . . . . . . . . . . . . . . . . 58

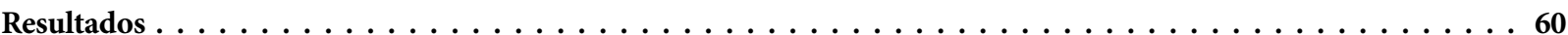

Avances del plan de proyecto de demostración de la tecnología de ultrasonido

en el marco del Programa Aguas LATU/Latitud . . . . . . . . . . . . . . . . . . . . . . . . . . . . 60
\end{abstract}

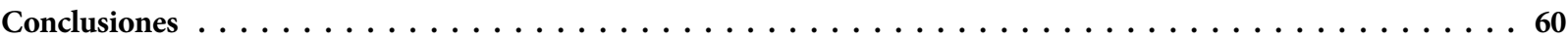

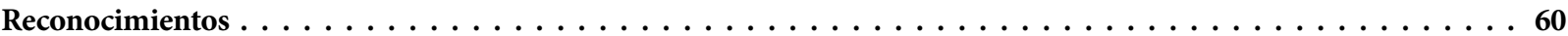

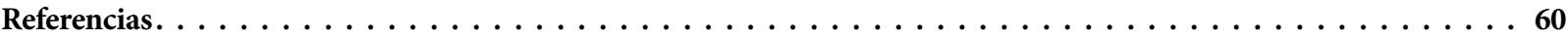

\section{Introducción}

Las cianobacterias o algas verde-azules se encuentran en muchos ecosistemas acuáticos. Las floraciones de cianobacterias nocivas pueden impartir malos sabores al agua o incluso contaminarla con toxinas. Estas floraciones consisten en el crecimiento excesivo dominado por una o múltiples especies y constituyen un peligro ambiental a causa de la posible liberación de cianotoxinas en ciertas condiciones ambientales (Chorus y Bartram, 1999). Las condiciones ambientales que pueden promover el crecimiento de cianobacterias y la producción de cianotoxinas son la concentración de nutrientes que cuando se encuentran en exceso promueven la eutrofización, la temperatura del agua, la intensidad lumínica, la salinidad, el movimiento del agua, etcétera. La incidencia de las floraciones de cianobacterias en aguas superficiales ha aumentado en las últimas décadas por el aporte excesivo de nutrientes debido a prácticas agrícolas intensivas, a los vertidos de aguas residuales y en muchos países al uso de fosfatos en detergentes (Kundu, et al., 2015), eventos posiblemente agravados por el cambio climático (Wells, et al., 2015).

Las cianotoxinas se producen principalmente en forma intracelular durante la fase de crecimiento exponencial. La liberación de toxinas en el agua puede ocurrir durante la muerte o senescencia celulares pero también puede ser debido a las circunstancias evolutivas derivadas o ambientalmente mediado como alelopatía o limitación de nutrientes relativamente repentina. Cuando las cianotoxinas se liberan después de la muerte celular lo hacen a través de flujo pasivo del contenido celular o de la célula lisada. En consecuencia, cuando existen floraciones de cianobacterias en recursos de agua potable, el tratamiento debería eliminar cianobacterias pero evitar la lisis celular y la liberación de toxina posterior de cianotoxinas al medio acuático (Merel, et al., 2013).

Las microcistinas, toxinas peptídicas cíclicas, son las más ampliamente distribuidas. La microcistina-LR es la variante más tóxica, pero hay un amplio rango que incluye además neurotoxinas y dermatotoxinas. Según la Agencia Ambiental de Estados Unidos, las floraciones de cianobacterias pueden ocasionar envenenamiento en ganado, peces y fauna silvestre (USEPA, 2015).

La exposición humana a cianobacterias y sus productos biológicamente activos es posible durante los deportes acuáticos y actividades de agua, o por la ingestión de agua contaminada. La Organización Mundial de la Salud (OMS, 2015) menciona los efectos en el ser humano, que incluyen irritaciones de la piel, los ojos, la boca y la nariz, dolor de estómago, de garganta, de cabeza, musculares, vómitos, fiebre, alergia, asma y urticaria, y afecciones de gravedad tales como hepatotoxicidad, nefrotoxicidad o efectos en el sistema nervioso. Estos efectos pueden ser de distinta gravedad por ingestión de las toxinas, por contacto dérmico o a través de diálisis. Así, por ejemplo, uno de los más graves casos de envenenamiento humano ocurrió en Brasil en 1996, cuando una floración de Microcystis en un depósito de agua de suministro contaminado con microcistinas en una clínica de tratamiento de hemodiálisis tuvo como resultado 56 muertes (Azevedo, et al., 2002). También hay un reporte de intoxicación aguda que requirió hospitalización por inmersión en una floración intensa existente en la represa de Salto Grande. La floración poseía un nivel de 48,6 $\mu \mathrm{g} / \mathrm{L}$ de microcistina-LR (Giannuzzi, et al., 2016).

Aunque la gran mayoría de productos dañinos de cianobacterias está confinada al interior de las células, estos finalmente se liberan en el agua circundante después de la muerte celular natural o artificialmente inducida. Hasta la fecha, más de 100 diferentes congéneres de microcistina han sido caracterizados y se nombran según el código de la letra de los aminoácidos incorporados en las posiciones dos y cuatro; por ejemplo, microcistina-LR contiene leucina $(\mathrm{L})$ en posición dos y arginina (R) en la posición cuatro. Las microcistinas se pueden introducir en los tejidos de los organismos a través 
de la dieta o por la ingestión de agua contaminada, luego de lo cual se acumulan en el hígado mediante el sistema de transporte de ácidos biliares, variando su toxicidad de acuerdo a la combinación de los aminonácidos en las dos posiciones variables del anillo peptídico (Schmidt, et al., 2014). Su toxicidad se manifiesta como un enlace covalente que se forma entre la toxina y las fosfatasas proteicas, especialmente en los hepatocitos, conduciendo a daños estructurales en las células y enfermedad hepática y del riñón.

Estas sustancias han sido clasificadas como posibles carcinógenos para órganos blancos: hígado y colon pertenecientes al Grupo 2B por la Agencia Internacional de Investigación del Cáncer (IARC, 2010). Para el agua potable, la Organización Mundial de la Salud ha recomendado un valor guía provisional de $1 \mu \mathrm{g} / \mathrm{L}$ total de microcistina-LR en agua para el consumo (OMS, 2011).

Los niveles de nutrientes y la luz parecen ser factores limitantes en el control de la síntesis de microcistinas. La gran oxigenación que se produce en la superficie puede generar condiciones oxidantes para las células y algunas hipótesis indicarían que las microcistinas actuarían como antioxidantes. Además de Microcystis spp., hay especies tales como Aphazimenon spp. que producen variadas cianotoxinas: cilindrospermopsina, anatoxina-a, saxitoxina y beta-metilamino-L-alanina, (BMAA) (USEPA, 2015). La BMAA es una toxina que también es producida por otras especies de cianobacterias tales como Nostoc y se ha vinculado a la generación de procesos neurodegenerativos tales como los de la enfermedad de Alzheimer, evidenciados por la posible relación causa efecto en la población Chamorro de la isla de Guam, quienes podrían tener contacto con la toxina a través de la dieta, ya que las plantas cícadas bioacumulan esa toxina en sus semillas por la existencia de Nostoc en simbiosis con sus raíces. Los murciélagos Fanihi (Pteropus mariannus mariannus, Pteropodidae) consumen esas semillas y bioacumulan la toxina en sus tejidos y, a su vez, estos animales son consumidos por los pobladores, lo cual sería una posible ruta de intoxicación, además de la ingestión de recetas que tienen en su preparación harina de estas semillas (Banack, et al., 2006). En Norteamérica se detectó una correlación entre los casos de Alzheimer, esclerosis lateral amiotrófica con el BMAA presente en tejido cerebral analizado post mortem en pacientes afectados (Pablo, et al., 2009).

Como reseñan Harke et al. (2016), tradicionalmente las reducciones de los aportes de $\mathrm{P}$ han sido el foco para el control de las floraciones cianobacterianas basadas en la premisa de que pueden cumplirse fuentes de $\mathrm{N}$ por fijación de nitrógeno. Como Microcystis es incapaz de abastecer sus requerimientos de $\mathrm{N}$ por fijación de $\mathrm{N}_{2}$, el crecimiento y la proliferación de Microcystis dependen exclusivamente de fuentes externas de N generadas por diversas actividades humanas, cuya ocurrencia natural puede ser aumentada marcadamente, incluyendo la agricultura, la urbanización y la contaminación industrial, o debido a la regeneración interna de formas combinadas de $\mathrm{N}$, principalmente como amonio. De modo que la proliferación mundial de Microcystis se encuentra relacionada a aumentos tanto de $\mathrm{P}$ como de $\mathrm{N}$ debido a la expansión de las actividades humanas. Esto, combinado con el hecho de que la mayoría de los sistemas eutróficos son muy turbios, lo que ocasiona una potencial limitación de la penetración de la luz, favorece las floraciones de cianobacterias. Estas pueden regular su flotabilidad y, o bien migrar verticalmente, hundiéndose para acceder a las aguas del fondo ricas en nutrientes, o bien flotar en forma de floraciones superficiales, optimizando así la utilización de energía radiante.

\section{Prevalencia mundial \\ de las floraciones de cianobacterias nocivas y de los brotes de toxicidad}

Las floraciones de cianobacterias tóxicas son eventos que ocurren cada vez más comúnmente en cuerpos de agua en todo el mundo, especialmente en las zonas templadas o tropicales, debido a la industrialización y a la agricultura intensiva. Se debe seguir investigando cómo se afectarán por el cambio climático las condiciones en cuanto a ingresos de fósforo y de nitrógeno hacia las aguas costeras y las cuencas y si esto produciría una exacerbación de las floraciones (Paerl, 2016). Una de las cianobacterias formadoras de floración más generalizadas en ecosistemas de agua dulce es Microcystis spp., la que sobrevive al invierno en el bentos y durante el verano se eleva hasta el epilimnion, donde puede acumularse y forma floraciones y espumas en la superficie del agua.

En los países desarrollados el problema de las floraciones de cianobacterias nocivas es aún de mayor magnitud que en nuestra región. Por ejemplo, en Estados Unidos el Gobierno Federal informó en el año 2008 sobre un plan de gestión que incluía también los impactos económicos y socioculturales. Además, comprendía un relevamiento de acciones de monitoreo, predicción y control. También recomendaba una gestión de respuesta con alto grado de coordinación entre investigadores, gestores, tanto de salud pública como ambientales, y agencias federales y establecía incentivos económicos (López, et al., 2008). Aun así, en agosto de 2014 un evento de floración dejó a la población de la ciudad de Toledo, Ohio, sin agua potable durante varios días, debido al nivel de cianotoxinas detectadas en ella, lo cual, según entienden Ho y Mi (2015), debería motivar el desarrollo de modelos estadísticos o mecanísticos para entender mejor los factores causales. Este evento dio por resultado la promulgación de decretos presidenciales que mandataron a la Agencia Ambiental EPA a tomar acciones aún más enérgicas y límites más estrictos para cianotoxinas en agua, con niveles diferenciales más exigentes para niños.

\section{Antecedentes de eutrofización y floraciones de algas y cianobacterias nocivas en Uruguay}

En nuestro país, el Proyecto Algas financiado por LATU a través de convocatoria abierta evaluada por ANII fue un proyecto interinstitucional entre Facultad de Ciencias, Instituto Clemente Estable y LATU. Entre sus resultados cuantificó los nutrientes del agua y los genes de las toxinas y las toxinas de algas y cianobacterias nocivas en el Río Uruguay y en el Río de la Plata, desde Salto a Punta del Este, generando además un protocolo de gestión (Kruk, et al., 2015). La situación en cuanto a eventos de floración algales ha sido cada vez más frecuente en los últimos tiempos y se han detectado muchos cuerpos de agua con esta problemática, especialmente durante la estación estival, lo que ha obligado a la empresa estatal potabilizadora, Obras Sanitarias del Estado (OSE), a tener que cambiar las tecnologías de potabilización que incluyen utilizar carbono activado y otros, y ha llevado al gobierno a decretar medidas de control y de gestión. 


\section{Métodos}

\section{Tratamientos para el control de cianobacterias y algas}

Las células se pueden eliminar mediante lisis limitada por procesos físicos como la clarificación o filtración de membrana y posterior remoción mecánica, pero también mediante agregado de sustancias químicas o por biomanipulación. En base a la información suministrada por la USEPA (2015) y otras fuentes bibliográficas señaladas, la siguiente es la descripción de los principales métodos existentes:

\section{Métodos físicos}

\section{Aireación}

Por medio del uso de aireadores que bombean aire a través de difusores se llevan las células a la superficie del agua. Es útil para pequeños estanques. Generalmente son más eficientes en aguas profundas.

\section{Manipulaciones hidrológicas}

El objetivo es interrumpir el proceso de estratificación de la columna de agua. Son fáciles de implementar en sistemas controlados (embalses, plantas de tratamiento), pero requieren volumen de agua suficiente como para controlar el caudal y pueden ser caros y afectar otros organismos.

\section{Mezcla mecánica}

Otro tratamiento de gran escala es usar la cavitación hidrodinámica mediante chorros a presión. De acuerdo a Wu et al. (2011), el tratamiento con un equipo llamado Sonolator (Sonic Corporation, USA) para tratar suspensiones de algas produjo un decrecimiento de $80 \%$ en su crecimiento. Se puede combinar con luz UV y filtros de fibra de vidrio, por ejemplo, para lograr tratamientos de agua para ciclos cerrados. Son mezcladores que llevan hacia abajo a las cianobacterias. Su uso está extendido en Estados Unidos, pero para pequeñas áreas.

Desecación

Se usa en embalses y consiste en desecar parte del agua y colocar nueva. Sus impactos ambientales pueden ser considerables.

Retiro de las espumas

Se usan equipos que extraen las espumas, en general junto con floculantes o coagulantes.

\section{Ultrasonido}

Se utiliza un equipo de irradiación de ultrasonido a frecuencias tales que destruyan la estructura celular interna de la cianobacteria al romper las vesículas de gas internas que usan para flotación. Es útil en pequeños estanques o tajamares. No utiliza sustancias químicas y suele no ser caro. Su efectividad depende de la geometría del cuerpo de agua y de las especies de cianobacterias. Se requiere mayor investigación. Se presenta más información sobre este método en el punto 2.3 .

\section{Métodos químicos}

Los antecedentes de tratamientos de control de algas usados a nivel internacional incluyen el agregado de aditivos químicos o biológicos para provocar su floculación (sulfato de cobre o de aluminio, paja de cebada envejecida, arcillas, o agregado de patógenos naturales tales como bacterias, virus y parásitos) (López, et al., 2008). Sin embargo, estos procedimientos pueden agregar compuestos nocivos para el medio ambiente o requerir mucho trabajo en su implementación. Incluyen alguicidas a base de cobre (sulfato de cobre, permanganato de potasio, cloro, cal, etcétera). Sus riesgos son lisar las células y dejar en libertad las toxinas, por lo que se suelen usar al comienzo de las floraciones. Algunos de ellos son también tóxicos para el zooplancton, otros invertebrados y los peces. Existen también experiencias con paja de cebada que, según se ha estudiado, produce sustancias alguistáticas durante su fermentación. Se ha utilizado en el Reino Unido con resultados positivos y puede producir mortandad de peces por la degradación de la materia orgánica.

\section{Coagulantes}

Los coagulantes se pueden usar para sedimentar las células en el fondo, donde no les llega la luz. En algunos casos pueden lisar las células y liberarse toxinas.

\section{Floculantes}

Los floculantes facilitan la sedimentación en el fondo anóxico, limitando los niveles de nutrientes.

\section{Oxígeno}

La oxigenación hipolimnética es una técnica que introduce un exceso de oxígeno para evitar que se liberen los nutrientes desde el sedimento, pero es relativamente cara. Gerling et al. (2014) estudiaron la aplicabilidad de esta tecnología y concluyeron que es adecuada para tratar cuerpos de agua poco profundos.

\section{Métodos biológicos \\ (biomanipulación y biodegradación)}

\section{Humedales flotantes artificiales}

Las plantas flotantes consumen los nutrientes y luego se cosechan. El método se puede implementar en pequeños cuerpos de agua pero tiene éxito limitado.

\section{Presión de pastoreo aumentada}

Este método incluye la remoción de peces que comen plancton o la introducción de zooplancton. Es menos agresivo ambientalmente que otros, pero podría afectarse la biodiversidad y requiere monitoreo continuo. Es adecuado para aguas poco profundas. También se puede incrementar la competición por recursos introduciendo otros productores primarios tales como macrofitas, para limitar el fósforo disponible y el crecimiento de las cianobacterias.

Un retiro suficientemente extenso de peces que comen plancton y bentos fue considerado por Søndergaard et al. (2008) como una herramienta eficaz para crear agua clara; sin embargo, podría requerirse repetir el procedimiento para obtener efectos a largo plazo en lagos ricos en nutrientes. Un efecto secundario importante de biomanipulación de lagunas es la disminución de las concentraciones de fósforo y nitrógeno que en sus estudios disminuyó sus concentraciones en cerca del $50 \%$ de los niveles iniciales.

\section{Tratamientos de remoción de las cianotoxinas}

Los efectos de microcistinas y otras cianotoxinas sobre la salud requieren métodos de tratamiento eficaces para su eliminación en agua de consumo. Se las puede remover por adsorción en carbón activado, por ósmosis inversa, o por oxidación química, a través de la ozonización o cloración. En Estados Unidos se observó que la remoción de las células de cianobacterias era muy buena en plantas de potabilización pero que las del tipo filamentoso, especialmente Aphanizomenon, podrían eventualmente pasar los filtros y 
encontrarse en agua potable (Szlag, et al., 2015). Los métodos de membranas de ultrafiltración y de nanofiltración y los de oxidación avanzada son promisorios ( $\mathrm{He}$, et al., 2016). La biodegradación de las microcistinas ocurre en condiciones aerobias en aguas superficiales, filtros de arena y efluentes cloacales. Por ejemplo, existen bacterias tales como una cepa de Sphingomonas sp. que degrada la microcistina-LR usando tres enzimas, incluyendo una microcistinasa.

\section{Características de la tecnología de ultrasonido y sus mecanismos de acción}

Según describe Dehghani (2016), las ondas ultrasónicas causan un gradiente de presión, cavitación y burbujas que por la vibración alcanzan un cierto diámetro a longitudes de onda ultrasónicas ( $6 \mu \mathrm{M}$ de diámetro, $1 \mathrm{MHz}$ frecuencia) y causan resonancia y mayores amplitudes de oscilación. Además de las grandes variaciones de presión en las burbujas, se producirían radicales libres. Se produce cavitación estable y cavitación de transición que solamente ocurre a altas energías de ultrasonido. Los efectos del ultrasonido en el crecimiento de las células dependen del tiempo, la frecuencia y la potencia. A alta frecuencia $(1.7 \mathrm{MHz})$ se producen radicales libres de alta potencia, causando daños estructurales en las células y en la clorofila-a. A bajas frecuencias $(20 \mathrm{kHz})$ y baja potencia también tiene como resultado la destrucción de células. Los radicales hidroxilos juegan un papel importante en la degradación de las toxinas tras la irradiación por ultrasonido.

Ha sido afirmado por proveedores de equipos que las paredes celulares de las algas no se rompen por esta tecnología, sino que se dañan solamente las internas, de modo que no se liberan inmediatamente las toxinas. De acuerdo a Kurokawa et al. (2016), la ruptura de las células depende de la frecuencia; la cavitación, más que los efectos directos del ultrasonido, es responsable de la ruptura de las algas, incluso en mayor medida que los efectos químicos causados por el ultrasonido. Kurokawa et al. postulan como posibilidad que influya el tamaño de la burbuja oscilante causada por la cavitación, pero también proponen que la ruptura se deba al efecto de la resonancia mecánica de las células algales. Rajasekhar et al. (2012) revisaron los efectos del ultrasonido en las cianobacterias y lo describieron como un proceso que consiste en la ruptura de las vacuolas de gas que les proveen flotación y que logran ubicarlas de tal forma que reciban la luz solar, lo cual regula su crecimiento.

Como mencionan Dunn y Pond (1978), ya en 1944 Lehmann describió el efecto del ultrasonido en la permeabilidad de las células, y en 1971 el mismo autor estudió la cinética de colapsado de estas vacuolas por ultrasonido, lo cual inspiró la tecnología. Por ejemplo, exponiéndolas a $120 \mathrm{~W}$ de potencia y $28 \mathrm{kHz}$ durante 3 segundos, el $80 \%$ de una suspensión de algas sedimentaba después de 30 segundos. En estudios experimentales la microscopía de transmisión mostraba que las vacuolas se recuperaban y quedaban intactas tal como antes de haber sido colapsadas.

Yamamoto et al. (2015) demostraron que la eficiencia del ultrasonido en suspensiones dependía tanto de la frecuencia como de la potencia acústica, trabajando entre 20 y 1146 $\mathrm{kHz}$ y distintas potencias, pero las mismas dependían de las propiedades mecánicas de las células de cada especie. Esto también ocurría a menores frecuencias e intensidades, según otros autores $\left(1.7 \mathrm{MHz} ; 0.6 \mathrm{~W} / \mathrm{cm}^{2}\right)$. En estas condiciones, hay cambios de conductividad eléctrica como indicador in- directo de la cavitación en suspensiones de algas, ya que se experimenta un aumento brusco de la conductividad de $M$. aeruginosa, lo cual sustenta la teoría de la resonancia de las vacuolas. Las fuerzas aplicadas a través de las ondas sonoras inducen vibraciones en las burbujas, las cuales pueden resonar a una frecuencia característica y determinada por las dimensiones de la vesícula y las propiedades del medio.

A diferencia de otras bacterias, las cianobacterias poseen clorofila, la cual absorbe luz tanto a $430-440 \mathrm{~nm}$ como a 660-681 nm, y otros pigmentos, tales como ficobilisomas que absorben a $470-650 \mathrm{~nm}$, lo cual los hace organismos fotosintéticos. Es por eso que se usa el monitoreo de los cambios de clorofila para medir la actividad fotosintética. Sin embargo, a diferencia de lo que ocurre con las plantas y las algas verdes, en las cuales hay una antena PSII y un complejo cosechador de luz hidrofóbico dentro de la membrana, ésta está ausente en cianobacterias y es reemplazada por un complejo extracelular soluble, el ficobilisoma compuesto por ficobiliproteínas. La foto-protección ante la sobre-excitación de los centros fotoquímicos en este caso puede obtenerse por disipación de la energía de excitación inducida por la proteína naranja carotenoide a nivel del ficobilisoma, que oficia así de "antena de las cianobacterias" (Kirilovsky y Kerfeld, 2012). Por otra parte, a diferencia de las algas verdes, las cianobacterias poseen ficocianina, la cual sufre mayores daños que la clorofila, afectándolas más que a las algas verdes.

Otras teorías apuntan también que la destrucción de las células de cianobacterias se produce por daños ocasionados por radicales libres por peroxidación de los lípidos, pero la causa principal es la pérdida de flotación. También hay efectos en la división celular y la morfología. M. aeruginosa es más sensible a la sonicación en el ciclo tardío de luz, o antes del atardecer y en la fase exponencial de crecimiento. Las algas filamentosas Anabaena circinalis y Aphanizomenon son aún más inhibidas que las unicelulares, pero por disrupción de la estructura filamentosa. La frecuencia está en un rango de 20 $\mathrm{kHz}$ a 1,7 MHz. Para seleccionar los parámetros hay que considerar frecuencias y potencias, el tiempo de exposición y el uso del agua y la especie que se quiere eliminar. Estos mismos autores ofrecen una tabla con las distintas combinaciones de estos parámetros y una revisión de métodos alternativos de control de cianobacterias en combinación con ultrasonido.

\section{Equipos comercialmente disponibles y aplicabilidad a ríos, lagunas, tajamares y estanques}

La tecnología está madura para la aplicación en embalses, lagunas y estanques. Según los fabricantes, el tratamiento es amigable para el medio ambiente y eficiente en cuanto a costos. Una boya auto-energizada se localiza en puntos seleccionados del lago. Su sistema acústico se programa en forma remota y genera frecuencias que resuenan a las células de las cianobacterias y las rompen (http://www.clearwater-pmpc. $\mathrm{com} /$ ). Como resultado de este proyecto se logró desarrollar el sistema de control de algas que usa ultrasonido, pero más bajo en costos de operación e instalación que otros, ya que el sistema es autónomo porque monitorea e interpreta los parámetros de calidad de agua.

El equipo diseñado por la empresa LG Sonic emite señales de ultrasonido basadas en el tipo de algas presentes y no afectaría a plantas, insectos u otras formas de vida presentes 
en el cuerpo de agua. Los paneles solares y baterías les proporcionan energía para operación continua y sus sensores están conectados a brazos sumergibles. Uno de sus modelos permite tratar superficies de agua con una cobertura de hasta $500 \mathrm{~m}$ en diámetro y en dos o tres semanas se trataría un tajamar mediano, pero eso se debe determinar experimentalmente en cada caso. Este dispositivo no solo afecta la flotación y fotosíntesis, sino que también impide la adherencia de las bacterias a las superficies, debilitando las bio-películas o biofilms, previniendo su formación (http://www.lgsonic.com/ how-does-lg-sonic-work/). Se lo suele usar para acuacultivo, campos de golf y tajamares de riego. Para su aplicación en ríos se encuentra todavía en escala experimental. Otras marcas comerciales provienen de India (http://m.indiamart.com/ tanclean-india/aboutus.html), Reino Unido, Nueva Zelandia, Australia (http://www.envirosonic-algaecontrol.com/), Japón (Honda Electronics Co. Ltd., Toyohashi, Japón).

\section{Otros usos del sistema de ultrasonido en el campo ambiental}

El ultrasonido de alta frecuencia se ha utilizado exitosamente para optimizar los tratamientos para aguas residuales de alta carga orgánica (Zinadini, et al., 2015). A frecuencias de 1-3 MHz el ultrasonido se puede emplear para cosechar microalgas, en tanto si es de alta intensidad $(20-50 \mathrm{kHz})$ activa las catálisis heterogéneas y enzimáticas de reacción con la biomasa, ayudando a obtener mayores rendimientos y reducir el tiempo de extracción en la producción de biocombustibles (Luo, et al., 2014). Este mismo autor recomienda que se seleccionen cuidadosamente los parámetros operacionales para aplicar correctamente esta tecnología para estos fines. En tanto, el ultrasonido pulsado se ha usado para bajar la carga microbiana de aguas naturales (Al-Juboori, et al., 2015).

\section{Probabilidad de impactos negativos}

El uso de ultrasonido en actividades diagnósticas clínicas es común desde hace varias décadas. O'Brien (1978) discute los efectos en tejidos biológicos de distintos tipos de ultrasonido y concluye que la evaluación de riesgos de la seguridad del ultrasonido para el ser humano es una cuestión de umbrales.

La selectividad del método en cianobacterias fue demostrada por Rajasekhar et al. (2012), comparando M. aeruginosa y otras algas verdes tales como Chlorella spp., siendo estas últimas poco afectadas por el ultrasonido. Esto es positivo, ya que son sustento de peces y otros organismos acuáticos. Como también ataca algas filamentosas y diatomeas, que forman obstrucciones de filtros en plantas de tratamiento, $y$ las de Anabaena spp., que causan olores y sabores, también sería positivo en este sentido. Esta menor diversidad no afectaría negativamente el ambiente porque se garantiza la producción primaria del ecosistema al existir algas verdes. Todos los otros organismos acuáticos no serían afectados por el ultrasonido. Según estos investigadores, la aplicación de ultrasonido no resulta ser nociva para el resto de la vida acuática ni destruye los organelos celulares de plantas más complejas. Las toxinas son liberadas por las cianobacterias al descomponerse, pero dejan de producirse al ir muriendo las células y como la reproducción de las algas disminuye, también lo hacen las toxinas liberadas. Las bacterias que descomponen nutrientes pueden entonces actuar.
En cuanto a los efectos en crustáceos, en estudios de laboratorio se han evidenciado efectos, según Lürling y Tolman (2014). A altas frecuencias, Holm et al. (2008) lo han usado para control de especies nocivas para aguas de proceso. De modo que para no agredir a las especies acuáticas en cursos de agua las intensidades y frecuencias deberán ser controladas cuidadosamente.

\section{Antecedentes de uso en el mundo: estudios de caso de aplicación piloto o a gran escala}

Un ejemplo de proyecto internacional de la aplicación de ultrasonido de bajas amplitudes colocado en columnas sumergidas que tienen mezcladores que llevan el agua a pasar por el tratamiento y se usan para control de floraciones algales es el de la Universidad de Adelaida, Australia, en colaboración con empresas que están investigando la eficiencia de esta tecnología (University of Adelaide, 2010). En Europa, el desarrollo del equipo LG Sonic para uso en lagos fue financiado a través del Programa Marco FP7-SME, bajo el nombre de proyecto de referencia 286875, titulado CLEARWATERPMPC ("Development of an efficient environmentally-friendly Algae Control System, based on ultrasound technology, designed for use in bigger ponds and lakes") desde 1/1/2012 con una duración de dos años (CORDIS, s.d.).

En su reseña Wu et al. (2011) discuten la aplicación a estudios piloto y en grandes lagos, tales como el Lago Senba, de Japón, con un volumen de $365000 \mathrm{~m}^{3}$ y profundidad media de $1 \mathrm{~m}$, en el que se emplearon 10 sistemas flotantes ultrasónicos y que operó satisfactoriamente a $200 \mathrm{kHz}$ de frecuencia (intensidad $0.1 \mathrm{~W} / \mathrm{cm}^{3}$ ). En este estudio el ultrasonido disminuyó la concentración de clorofila-a en el verano. Este proceso se ha combinado también con bombas de agua en pequeños estanques, para luego expandir el agua sonicada hacia la superficie de un tanque de $9000 \mathrm{~m}^{3}$. En China, la Universidad del Sureste empleó un enfoque diferente para disminuir las células. En un cerramiento de $400 \mathrm{~m}^{2}$ del Lago Taihu, usaron un bote con equipo ultrasónico (20 sondas) y transductores de a bordo.

En proyectos realizados en Estados Unidos por Kaser y Perdue (2015) en el Lago Rockwell para controlar las algas antes de entrar en la planta de tratamiento de agua se usó el sistema compuesto por equipo de ultrasonido con sensores de parámetros de calidad de agua colocados en boya con sondas de $\mathrm{pH}$, turbiedad, sólidos suspendidos, clorofila-a (algas), ficocianina, oxígeno disuelto y temperatura. Con base en estos parámetros el sistema cambia el patrón de vibración. Cada boya está equipada por paneles solares para autoabastecerse de energía. El batallón de ingenieros de la Armada de Estados Unidos evaluó este método y describe su aplicación a un club campestre de Rockland, Nueva York. Este sistema también fue probado en Canoe Brook, un embalse en Nueva Jersey, y se concluyó que se redujeron los problemas de sabor y olor y de niveles de algas que entraban en la planta potabilizadora, requiriendo menos alúmina para tratarla, dando un resultado económico de 1.8 años de repago. En Corea del Norte se logró reducir los aportes de nutrientes pero, aun así, se evidenciaron eventos de eutrofización en los tramos inferiores del Río Nakdong, por lo cual, para disminuir la cantidad de fósforo en el agua y en forma simultánea en el fondo del río se aplicaron tecnologías para suministrar oxígeno disuelto al sedimento 
para remover nutrientes, lo que hizo menos económico el procedimiento (Tekile, et al., 2015).

\section{Resultados}

\section{Avances del plan de proyecto de demostración de la tecnología de ultrasonido en el marco del Programa Aguas LATU/Latitud}

Dado que el método de ultrasonido es considerado como promisorio pero aún requiere investigación para evaluar su efectividad e inocuidad, actualmente estamos demostrando esta tecnología en un proyecto colaborativo en el Centro Regional Sur de la Facultad de Agronomía de la Universidad de la República que tiene 230 vacas en un tambo experimental. Existe allí un tajamar eutrofizado al que expusimos a un tratamiento con un equipo de ultrasonido LG-Sonic E line, observando que la concentración de clorofila-a descendió de 766 a $159 \mu \mathrm{g} / \mathrm{L}$ en el plazo entre 25/6/2015 a $2 / 7 / 2015$, pero se requieren mayores trabajos de validación para conseguir resultados estadísticamente significativos y para contrarrestar los efectos de las condiciones ambientales variables. Para ello, continuamos evaluando tanto las comunidades de peces, de bentos y de zoo y fitoplancton, como la calidad del agua, utilizando métodos físicos y químicos. Los estudios de ecotoxicidad dispuestos abarcan desde efectos agudos, crónicos, mutagénicos y de neurodegeneración, a la aplicación de técnicas de biología molecular y metagenómica. En pasos subsiguientes se realizarán estudios de los efectos en el ecosistema, realizando cerramientos en el tajamar en la zona de influencia del equipo para tener más acotado el sistema.

\section{Conclusiones}

Las metodologías existentes para control de floraciones de algas y cianobacterias nocivas son variadas, pero hasta el momento no existe ninguna totalmente satisfactoria. Es por ello que es necesario continuar investigando y sopesando los beneficios y los riesgos de cada una, tanto económicos, como ambientales. En el caso del sistema por irradiación de ultrasonido la extensión cada vez mayor de esta tecnología a nivel mundial la hace particularmente atractiva, pero la gestión de las floraciones tiene que involucrar un control muy estrecho de los equipos y de los otros usos del agua además de los requeridos para potabilización, tales como los recreativos. Sin perjuicio de estas soluciones de remediación, la prevención por disminución de los aportes de nutrientes y la educación ambiental siguen siendo clave para realizar una buena gestión de los recursos hídricos y evitar tener que recurrir a medidas paliativas o de mitigación de los daños realizados sobre los ecosistemas acuáticos.

\section{Reconocimientos}

Las investigaciones se vienen realizando actualmente en forma de proyectos colaborativos en el marco del Programa Aguas LATU y Latitud con una red nacional e internacional. En el proyecto de restauración de lagunas trabajamos en estrecha colaboración con la Facultad de Agronomía de la Universidad de la República (UdelaR). También colaboraron el Instituto Pasteur y en algunas etapas la Dirección Nacional de Medio Ambiente. En las fases de demostración de la eficiencia e inocuidad de otros procedimientos de restauración de lagos se realizarán proyectos de investigación académica aplicada con la Facultad de Ciencias de UdelaR y la Universidad Wageningen, de Holanda. En las fases de tratamientos de efluentes de los tambos para control de las descargas puntuales participan UNESCO-IHE, Delft, Holanda y Univalle, Colombia. Los efectos tóxicos neurodegenerativos y otros están siendo evaluados junto con el Instituto Pasteur de Montevideo, ose, el Instituto Clemente Estable, la Universidad ORT, la Universidad del Trabajo (UTU) y la Universidad Tecnológica (UTEC). La autora agradece el aporte conceptual del Prof. Daniel Dietrich de la Universidad de Konstanz, Alemania en cuanto a aspectos de la toxicidad neurodegenerativa de las cianotoxinas.

\section{Referencias}

Azevedo, S., Carmichael, W., Jochimsen, E., Rinehart, K., Lau, S., Shaw, G. y Eaglesham, G., 2002. Human intoxication by microcystins during renal dialysis treatment in CaruaruBrazil. En: Toxicology, 181-182, pp.441-446.

Al-Juboori, R. A., Aravinthan, V., y Yusaf, T., 2015. Impact of pulsed ultrasound on bacteria reduction of natural waters. En: Ultrasonics Sonochemistry, 27, pp.137-147.

Banack, S. A., Murch, S. J. y Cox, P. A., 2006. Neurotoxic flying foxes as dietary items for the Chamorro people, Marianas Islands. En: Journal of Ethnopharmacology, 106, pp.97-104

Chorus, I., Bartram. J., ed., 1999. Toxic cyanobacteria in water: a guide to their public health consequences, monitoring and management. London: E\&FN Spon.

CORDIS, s.d. Final report summary - CLEARWATERPMPC (Development of an efficient environmentally-friendly Algae Control System, based on ultrasound technology, designed for use in bigger ponds and lakes) [En línea]. [s.l.]: CORDIS.[Consulta: noviembre de 2016]. Disponible en: http://cordis.europa.eu/result/rcn/156461_en.html

Dehghani, M. H., 2016. Removal of cyanobacterial and algal cells from water by ultrasonic waves - A review. En: Journal of Molecular Liquids, 222, pp.1109-1114.

Dunn, F. y Pond, J.B., 1978. Selected non-thermal mechanisms of interaction of ultrasound and biological media. Chap. IX. En: Fry, Francis J., ed., 1978. Ultrasound: its application in medicine and biology. New York: Elsevier.

Gerling, A. B., Browne, R.G.,Gantzer, P.A., Mobley, M.H., Little, J.C. y Carey, C.C., 2014. First report of the successful operation of a side stream supersaturation hypolimnetic oxygenation system in a eutrophic, shallow reservoir. En: Water Resources, 67, pp.129-143

Giannuzzi, L., Sedan, D., Echenique, R. y Andrinolo, D., 2011. An acute case of intoxication with cyanobacteria and cyanotoxins in recreational water in Salto Grande Dam, Argentina. En: Marine Drugs, 9(11), pp. 2164-2175.

He, X., Liu, Y.-L., Conklin, A., Westrick, J., Weavers, L. K., Dionysiou, D. D, Lenhart, J.J., Mouser, P.J., Szlag, D. y Walker, H.W., 2016. Toxic cyanobacteria and drinking water: Impacts, detection, and treatment. En: Harmful Algae, 54, pp.174-193.

Holm, E.R., Stamper, D.M., Brizzolara, R.A., Barnes, L., Deamer, N. y Burkholder, J.M., 2008. Sonication of 
bacteria, phytoplankton and zooplankton: Application to treatment of ballast water. En: Marine Pollution Bulletin, 56, pp.1201-1208.

International Centre for Research on Cancer, [s.d]. IARC monographs on the evaluation of carcinogenic risks to humans. List of classifications [En línea]. Lyon: IARC. [Consulta: 18 de noviembre de 2016]. Disponible en: http://monographs.iarc.fr/ENG/Classification/latest_ classif.php.

Kaser, M. y Perdue, A., 2015. Utilizing ultrasonic technology to manage algal blooms in Lake Rockwell. En: Honors Research Projects, Paper 68.

Kruk, C., Piccini, C., Segura, A., Nogueira, L., Carballo, C., Martínez De La Escalera Siri, G., Calliari, D., Ferrari, G., Simoens, M., Cea, J., Alcántara, I., Vico, P. y Míguez, D., 2015. Herramientas para el monitoreo y sistema de alerta de floraciones de cianobacterias nocivas: Río Uruguay y Río de la Plata. En: INNOTEC, 10, pp. 23-39.

Kundu, S., Coumar, M., Rajendiran, S. y Ajay, Rao, A., 2015. Phosphates from detergents and eutrophication of surface water ecosystem in India. En: Current Science, 108(7), pp.1320-1325.

López, C.B., Jewett, E.B., Dortch, Q., Walton, B.T. y Hudnell, H.K., 2008. Scientific assessment of freshwater harmful algal blooms. Washington: Interagency Working Group on Harmful Algal Blooms, Hypoxia, and Human Health of the Joint Subcommittee on Ocean Science and Technology.

Assessment of Freshwater Harmful Algal Blooms. En Interagency Working Group on Harmful Algal Blooms, Hypoxia, and Human Health of the Joint Subcommittee on Ocean Science and Technology. Washington, DC.

Luo, J., Fang, Z. y Smith, R. L., 2014. Ultrasound-enhanced conversion of biomass to biofuels. En: Progress in Energy and Combustion Science, 41, pp.56-93.

Lürling, M. y Tolman, Y., 2014. Beating the blues: Is there any music in fighting cyanobacteria with ultrasound? En: Water Resources, 66, pp. 361-373.

Merel, S., Walker, D., Chicana, R., Snyder, S., Baurès, E. y Thomas, O., 2013. State of knowledge and concerns on cyanobacterial blooms and cyanotoxins. En: Environment International, 59, pp.303-327.

American National Standards Institute, 1988. NSF/ANSI 61: Drinking water system components. Michigan: NSF.

O'Brien Jr, W.D., 1978. Safety of ultrasound. En: deVlieger, M., et al., eds. Clinical handbook of ultrasound. New York: Wiley.

OMS, 2011. Guías para la calidad del agua potable [En línea]. $3^{\mathrm{a}}$ ed. Ginebra: OMS. [Consulta: 18 de noviembre de 2016]. Disponible en: http://www.who.int/water sanitation_health/dwq/gdwq3rev/es/

OMS, 2015. Water-related diseases. Cyanotoxins [En línea]. Ginebra: OMS. [Consulta: 11 de junio de 2015]. Disponible en: http://www.who.int/water_sanitation_ health/diseases/cyanobacteria/en/

Pablo, J., Banack, S., Cox, P., Johnson, T., Papapetropoulos, S., Bradley, W., Buck, A. y Mash, D., 2009. Cyanobacterial neurotoxin BMAA in ALS and Alzheimer's disease. En: Acta Neurologica Scandinavica, 120(4), pp. 216-225.

Paerl, H. W., Gardner, W. S., Havens, K. E., Joyner, A. R., McCarthy, M. J., Newell, S. E., Qing, B. y Scott, J.T., 2016. Mitigating cyanobacterial harmful algal blooms in aquatic ecosystems impacted by climate change and anthropogenic nutrients. En: Harmful Algae, 54, pp. 213-222.

Rajasekhar, P., Fan, L., Nguyen, T. y Roddick, F.A., 2012. A review of the use of sonication to control cyanobacterial blooms. En: Water Research, 46(14), pp.4319-4329.

Schmidt, J. R., Wilhelm, S. W. y Boyer, G. L., 2014. The fate of microcystins in the environment and challenges for monitoring. En: Toxins, 6(12), pp.3354-3387. http://doi. org/10.3390/toxins6123354

Søndergaard, M., Liboriussen, L., Pedersen, A.R. y Jeppesen, E., 2008. Lake restoration by fish removal: short-and long-term effects in 36 danish lakes. En: Ecosystems, 11, pp.1291-1305.

Szlag, D. C., Sinclair, J. L., Southwell, B. y Westrick, J. A., 2015. Cyanobacteria and cyanotoxins occurrence and removal from five high-risk conventional treatment drinking water plants. En: Toxins, 7(6), pp.2198-2220. http://doi. org/10.3390/toxins7062198.

Tekile, A., Kim, I. y Kim, J., 2015. Mini-review on river eutrophication and bottom improvement techniques, with special emphasis on the Nakdong River. En: Journal of Environmental Sciences, 30, pp.113-121.

University of Adelaide, 2010. Using ultrasound to control toxic algal blooms [En línea]. Adelaide: University of Adelaide. [Consulta: noviembre de 2016]. Disponible en: https:// www.adelaide.edu.au/news/news40181.html

USEPA, 2015. Cyanobacteria/cyanotoxins. Nutrient policy and data [En línea]. Boston: USEPA. [Consulta: $11 \mathrm{de}$ noviembre de 2016]. Disponible en: http://www2.epa. gov/nutrient-policy-data/cyanobacteriacyanotoxins.

Virkutyte, J., 2015. 36 - The use of power ultrasound in biofuel production, bioremediation, and other applications. En: Power Ultrasonics, pp.1095-1122.

Wells, M. L., Trainer, V. L., Smayda, T. J., Karlson, B. S. O., Trick, C. G., Kudela, R. M. y Cochlan, W. P., 2015. Harmful algal blooms and climate change: Learning from the past and present to forecast the future. En: Harmful Algae, 49, pp.68-93.

Wu, X., Joyce, E.M. y Mason, T.J., 2011. The effects of ultrasound on cyanobacteria. En: Harmful algae, 10, pp.738-743.

Zinadini, S., Rahimi, M., Zinatizadeh, A. A. y Shaykhi Mehrabadi, Z., 2015. High frequency ultrasound-induced sequence batch reactor as a practical solution for high rate wastewater treatment. En: Journal of Environmental Chemical Engineering, 3(1), pp.217-226. 